\title{
El papel de las instituciones políticas en España (1980-2002): un estudio aplicado a las políticas detelecomunicación
}

OlgaGil *

\section{Introducción}

España se erige a finales del siglo xx entre uno de los siete países desarrollados que generan la mayoría de las exportaciones mundiales en el sector de servicios, junto con los Estados Unidos, Francia, Italia, Alemania, Gran Bretaña y Japón - sector para el que las telecomunicaciones son de máxima relevancia. Es más, España e Italia son los dos países que experimentan a fines de la década de 1990 el mayor crecimiento en las exportaciones relativas al sector servicios (M cEown 1999: 20). Teniendo en cuenta que las telecomunicaciones son un sector básico como precondición para la bonanza del sector servicios, y que una sola compañía, como Telefónica, aporta el 2 por 100 del valor añadido bruto de la economía españolal, se plantea la pregunta de cuál es el papel jugado por las instituciones políticas dentro del sector de las telecomunicaciones: ¿cómo se gobierna el sector? La respuesta a la pregunta anterior es el objetivo de este trabajo, en el que se va a mostrar cómo las instituciones políticas actúan sobre el mercado en las dos últimas décadas del siglo xx usando la estrategia del cambio institucional como medio para alterar el equilibrio político anteriormente existente - de manera democrática, pero con implicaciones para la caracterización del término- . Una pregunta abierta para investigaciones futuras es si esta estrategia es suficiente para devolver al Estado un papel predominante en un mundo donde las transacciones financieras y las empresas organizadas en red erosionan su papel como mediador en el contrato social.
Pudiera argumentarse que los cambios ocurridos en España en la regulación del sector a partir de 1980 son fruto de cambios de mayor calado, como consecuencia del cambio de sistema político 0 , lo que es lo mismo, de la transición de un régimen dictatorial a uno democrático. Existen dos evidencias que, aunque no invalidan la importancia de este hecho, cuestionan su capacidad determinante. En primer lugar, países con tradiciones de mocráticas asentadas como Gran Bretaña, Francia, Australia o N ueva Zelanda han seguido la pauta de poner en marcha cambios institucionales con el objeto de cambiar políticas sectoriales. En segundo lugar, existen mecanismos concretos y particulares, detallados en este estudio, que incentivan o blindan los posibles cambios, y estos mecanismos no están correlacionados con tipos de régimen específicos.

D entro del sector servicios, el sector español de las telecomunicaciones ha evolucionado desde un monopolio tradicional protegido por el Estado a un mercado más abierto, con espacio para varios competidores. En esta evolución el papel de las instituciones políticas ha sido fundamental. Con el objeto de romper las inercias que convierten al sector en generador de rentas para un solo actor monopolista, se opta desde distintos niveles de gobierno por estrategias basadas en el cambio institucional como medio para alterar el equilibrio político. El cambio institucional viene generado por el gobierno democrático y por las instituciones de la U nión Europea. Ambos niveles de gobierno consiguen abrir la corriente de rentas que el sector genera a nuevos actores sociales desde la década de 1980. A este objetivo de apertura contribuyen también las Comunidades Autónomas, cuyas actuaciones son relevantes (GIL, 2002). 
En este trabajo, en primer lugar, se aborda la teoría que permite analizar el caso a partir de presupuestos generalizables y parsimoniosos. En esta sección se comienza por especificar las hipótesis que dirigen la investigación, así como las definiciones de regulación, esferas y niveles de autoridad y cambio de políticas. A continuación se introduce el estudio empírico del caso español. Se finaliza con unas conclusiones sumarias.

\section{Teoría}

La hipótesis de partida de este trabajo afirma que dada la existencia de ciertas instituciones $(X)$ que hay que especificar, ocurre el cambio de políticas ( $Y$ ) bajo ciertas condiciones que son objeto de estudio empírico. La hipótesis de trabajo se pone a prueba para el caso español, antes y después de la entrada del país en la Unión Europea. El trabajo teórico, que se presenta a continuación, se centra en las definiciones básicas de los términos empleados.

\section{Regulación}

El término regulación se refiere a un abanico de políticas tales como los subsidios, las cuotas a las importaciones, las modalidades de empresa pública o privada, e incluso la creación de nuevos derechos de propiedad y de mercados específicos para intercambiarlos, siguiendo la definición dada por St I LER (1971: 4-7). La regulación se refiere a una forma de alterar el comportamiento de un mercado mediante la promulgación de reglas coactivas que gobiernan al gún aspecto de la producción, los atributos cualitativos, la entrada y/0 el precio al que un bien es adquirido o vendido por otros (N OLL, 1987: 465-468).

\section{Los niveles y esferas de autoridad, los tipos de reglas y los tipos de mercado}

M etodológicamente, a las instituciones reguladoras dentro del sector económico elegido se les llama «niveles» y «esferas» de autoridad. Los niveles de autoridad en este análisis se corresponden con los distintos niveles de gobierno que ejercen su autori- dad sobre un territorio, y en nuestro caso seleccionado, sobre un sector de políticas concreto. En el caso español, para el sector de las telecomunicaciones se encuentra el nivel del Estado central, al que se une prácticamente a la par el de la UE y el regional en la década de 1980. M etodológicamente también puede incluirse un nivel supranacional, como por ejemplo la O rganización M undial de Comercio (OM C), o bien el nivel local, y todos son niveles de autoridad. Sin embargo, la OM C resulta de interés limitado hasta que se hayan establecido reglas y precedentes para la resolución de disputas a nivel nacional (W AVERM AN y ESEN, 1997: 115).

En cuanto a las esferas de autoridad, se alude a instituciones, incluidas en los niveles de autoridad, que no se encuentran sólo sometidas a simples relaciones de jerarquía respecto de los niveles existentes. Son instituciones cuyas competencias son diversas y cuya autoridad puede revelarse clave, bien para paralizar a otras esferas o niveles de autoridad con su poder de veto, o bien para reforzar sus competencias2. Los tribunales como esfera de autoridad se diferencian del ejecutivo, el legislativo y otras agencias especializadas. Esta diferenciación no es de tipo jerárquico. La conveniencia de distinguir esferas de autoridad en los distintos niveles es necesaria para medir los efectos de su ausencia o existencia. También es necesaria para poder analizar el papel de las esferas de autoridad respecto de instituciones en el mismo nivel de políticas y en otros niveles.

Esta clasificación dista mucho de ser estática en el sentido de que los efectos tanto de niveles como de esferas de autoridad pueden estudiarse tomando en cuenta largos períodos de tiempo para llegar a resultados comparativos diacrónicos.

\section{Los tipos de mercado}

Partiendo de marcos teóricos corporatistas, CAw SON et al. (1990) recogen una tipología de los modos de influencia del Estado sobre los intereses económicos. Esta tipología sirve para interpretar la lucha competitiva que se desata en los mercados, partiendo de la teoría weberiana de la exclusión social. Según esta teoría, las relaciones entre empresas dentro de un mercado, «deben ser analizadas como relaciones de poder mediadas por el Estado» (C awson et al., 1990: 12). Las empresas buscan y encuentran apoyo en la coerción estatal, capaz de impedir la participación de otras empresas no beneficiadas en sectores del mercado. El Estado se convierte en árbitro, y en este proceso, se produce la revisión de patrones existentes de exclusión y privilegio (CAwson et al., 1990: 2). De este modo, se llega a una concepción en la que las empresas son actores políticos, además de económicos. Se convierten en actores políticos que buscan in- 
fluenciar las políticas públicas, ejercer su poder de mercado y re sistir o acomodarse al poder de mercado de otras empresas haciendo uso de sus relaciones con el Estado. Partiendo de esta concepción, la definición misma de mercado es el resultado de un «proceso político» (Cawson et al., 1990: 13).

En el campo de interés de este estudio, los sectores económicos, existen tres tipos de acción estratégica empresarial, orientadas a asegurar una posición dominante en el mercado, siguiendo a C Awso n et al. (1990: 22-24). Todas las acciones estratégicas tienen su base en la lógica de la exclusión/inclusión.

En primer lugar, se da la exclusión mediante el aseguramiento de una posición competitiva dominante frente a otros compe tidores. Ésta es una estrategia agresiva de las empresas, orientada al mercado, más que hacia las autoridades públicas. Bajo este tipo de exclusión no existe cooperación con otros productores. Sin embargo, son posibles los acercamientos en torno a intereses comunes, como la distribución o los estándares técnicos. Sin embargo, entre las empresas domina la hostilidad sobre las relaciones de «hermandad», en la terminología weberiana.

Dentro de la exclusión mediante estrategias competitivas pueden encontrase subcasos. Uno es el intento de asegurar mediante el éxito a través de la competencia una posición de monopolio en la producción. Los instrumentos que contribuyen al éxito son la entrada en un mercado con prioridad, y la introducción de nuevos productos o procesos. Una segunda forma de exclusión por estrategia competitiva se basa en asegurar privilegios sobre la innovación mediante patentes que fijan derechos de propiedad. La estandarización puede también ser usada como instrumento con el mismo objetivo.

En segundo lugar, existen mercados en los que predomina como lógica de exclusión la acción asociativa. Bajo esta lógica, los productores definen cual es su interés colectivo de forma que se evite la estrategia competitiva. Ésta es una estrategia de carte lización, aun sin llegar a constituirse como cartel. Tal estrategia se encuentra en la formación de asociaciones de comercio que proporcionan un marco organizativo para la percepción de intereses comunes. Cuando estas asociaciones son poderosas pueden ejercer sanciones contra miembros que violan acuerdos colectivos, imponer multas o expulsar miembros. Este fenómeno ha sido denominado «el gobierno del interés privado» por $M$ ax Weber (1978: 342).

«A pesar de que la competición continua vigente entre ellos, los competidores actúan juntos en forma de "grupo de interés" contra aquellos que no forman parte del grupo; existe una tendencia creciente a establecer algún tipo de asociación con regulaciones racionales; si el interés monopolístico persiste, llega el momento en el que los competidores... establecen un orden legal que limita la competición mediante monopolios formales... En tales casos, el grupo de interés se ha convertido en un "grupo legalmente privilegiado" y sus participantes en "miembros privilegiados". Este tipo de exclusión, como lo llamamos, es un proceso que se repite....”.

Un tipo especial de acción asociativa es aquel en el que participan actores económicos y sociales junto con agencias gubernamentales. Es el caso de las redes cooperativas (cooperative networks) en las que se desarrollan productos de tecnología punta como ha sido el caso en Estados U nidos con los semiconductores, circuitos integrados, ordenadores, telecomunicaciones por medio de microondas... Una de las ventajas de las redes cooperativas es la relación que establecen entre organizaciones con bases de conocimiento muy variadas. Como señala H O LLINSWORTH (1997: 142), este tipo de coordinación no es posible dentro de una jerarquía, ya que ninguna empresa cuenta con el conocimiento ni los recursos para desarrollar los productos antes citados. Como ejemplo de redes cooperativas se encuentran las relaciones entre empresas auspiciadas por el Estado, 0 un poder supranacional como el de la Unión Europea con fines de investigación y desarrollo.

El tercer tipo de exclusión es el que se realiza por medio del Estado. En este caso la organización económica busca la protección de su posición en el mercado mediante la aprobación de le yes que limitan a los competidores. Con esto logra deshacerse del coste de mantener su posición privilegiada, que recae en el erario público. Este caso se puede dar como resultado de la pre sión de la empresa o sector económico sobre el gobierno, o bien porque el Estado entienda que la empresa es representativa para llevar a cabo objetivos de gobierno. También puede ocurrir que el gobierno garantice la protección para un sector con fines electoralistas. Tales privilegios pueden ser posteriormente revocados por un gobierno de distinto signo en un proceso que beneficia a otras empresas. Las nuevas beneficiarias acceden a los privilegios mediante su «usurpación» en la terminología weberiana.

\section{Antecedentes y contexto: grupos excluidos en España y conflictos políticos (1877-2002)}

En España existe un primer período (1877-1924) de exclusión por medio de la competencia, en el que hay que cualificar que tal competencia no está garantizada por ningún marco institucional político ni social. Coexisten las compañías telefónicas de diversos tamaños y hay tensiones entre la nacionalización y la privatización de redes en solo meses o semanas, según el gobier- 
Tabla 1

Grupos excluidos y favorecidos en el sector del mercado de las telecomunicaciones español (1877-2002) en distintos periodos

\section{Exclusión por competencia (1877-1924)}

«Competencia» en la que predominan las bajas barreras de entrada en la industria a priori.

\section{Excluidos}

Aquellos sin relaciones clientelares, capital o tecnología para afrontar el negocio en caso de interés.

\section{Beneficiados}

Se potencian las telecomunicaciones con las plazas extrapeninsulares, como el norte de África, de interés defensivo e «ntegrador». Importancia de las comunicaciones estratégicas militares.

\section{Exclusión por medio del Estado (1924-1998)}

\section{Excluidos}

Los operadores antes existentes, expulsados del mercado.

El Estado y la burguesía financiera nacional no son capaces de imponer condiciones mas beneficiosas, ni al comienzo, ni en el medio plazo. En concreto, el contrato de la CTN E con el Estado relaciona innovación o adelantos tecnológicos con la declaración de servicio publico, con lo cual el monopolio privado goza de la mejor protección posible.

\section{Beneficiados}

El monopolio. El Estado en el corto plazo (mediante premios para el gobierno de turno).

Cuadros técnicos e ingenieros españoles que se forman durante estos años con el know-how americano. Desarrollo de un sector para el que no se contaba con suficiente capital en España.

\section{A partir de 1946}

Cambios en asignación de la corriente de rentas al nacionalizar las acciones de ITT. M ayor poder estatutario para la compañía, aunque el gobierno nombra al presidente y gran parte de los miembros del consejo de administración. Ingenieros, cuadros técnicos, mujeres - que pueden incorporarse como telefonistas- . Red de empleos cuasi funcionariales que pueden beneficiar a los afines al régimen de Franco

ITT sigue beneficiándose, ya que hasta los años 1960 el mantenimiento y la investigación están controlados por ella.

A partir de la década de 1960 se diversifica el panorama de empresas extranjeras proveedoras de CTNE.
Exclusión por medio dela competencia (1998-)

\section{Excluidos}

Tendencia a la perdida del control político directo del monopolio dada la pequeña participación estatal en la compañía por parte del Estado - que comienza en los años 1960 con la ampliación de las acciones a los pequeños ahorradores «las matildes», y finaliza con la privatización total de la compa ñía- . Así, Telefónica queda fuera del juego político, pero no partidario. Grupos importantes excluidos son aquellas empresas con tecnología punta que pudieran competir agresivamente con Telefónica en momentos clave.

\section{Beneficiados}

Usuarios empresariales (variedad de servicios, limitaciones a subsidios cruzados, paquetes descuento de las grandes operadoras). Este beneficio está siempre restringido por el factor clave de que las empresas con tecnología más avanzada entran más tarde en el mercado debido a la exclusión política y social que sobre ellas se practica.

Elaboración: Olga GIL y Juan José M Ontero. Fuentes: GIL (1994, 1995) y M ONTero (1999).

no de turno. En este clima de «competencia» las barreras de entrada en la industria son bajas a priori. Sin embargo, dado que las empresas operan a diversos niveles - municipio, provinciaprobablemente el clientelismo endémico de la época determina en gran parte el mecanismo de recepción de licencias.

En 1924 se suprime la competencia por razones de «defensa nacional y orden publico», y el Estado se erige en máximo garante de la prerrogativa de monopolio de una sola firma, la Compañía Telefónica N acional de España (CTNE). Esto deja en el me jor de los escenarios a la empresa monopolista. Por un lado, un mercado cerrado a competidores externos, o paralelos dentro de los confines nacionales, en el que a diferencia del estadounidense, no existe la amenaza de legislación anti-oligopolio 0 anti-monopolio. Se trata de un mercado cautivo y protegido para una compañía permeada por la influencia accionarial de la estadounidense ITT. Los mecanismos de monopolización usados en su momento son la no renovación de licencias a las compañías que habían de someterse a permisos de renovación periódicos, y la compra por parte de la CT NE de compañías comerciales acuciadas por la crónica de la no renovación anunciada.

La fase de exclusión por medio de la competencia que comienza en 1998 tiene sus antecedentes remotos en 1982, con el comienzo del resquebrajamiento del ejecutivo como regulador unitario, fruto de la Constitución española de 1978 y de los nuevos actores que aparecen con fuerza renovada, los tribunales, las Comunidades Autónomas y el nivel de autoridad de la Unión Europea desde 1986. Tanto el Tribunal de D efensa de la Competencia (1989) como la Comisión del Mercado de las Telecomunicaciones son instituciones de inspiración comunitaria con importancia en la ordenación de las telecomunicaciones.

La privatización de la casi totalidad de las acciones de la compañía es puesta en marcha por un gobierno de centro izquierda (PSOE), y la finaliza un gobierno de centro derecha (PP), que nombra al presidente de la misma, y diseña un marco 
de competencia que aún favorece a la compañía3. En octubre de 1995 la compañía Airtel se convierte en el primer competidor histórico de Telefónica, en el sector de la telefonía móvil4. A esta compañía se unirá más tarde Amena como tercer operador en el mismo subsector. En 1997, Retevisión, en consorcio con Endesa y la italiana Stet abren otra brecha en el monopolio de Telefónica, con una estrategia que busca atraer a los usuarios empresariales de telefonía fija y, en segundo lugar, a los domésticos. En 2002, sin embargo, sigue abierto el debate sobre la desagregación del bucle local, que haría posible la competencia a nivel de mercado metropolitano de telefonía fija, subsector en el que los nuevos operadores que se han ido incorporando aún han de negociar el acceso con Telefónica.

Cuando el Estado se deshace de sus participaciones directas y de su papel rector en el consejo de administración de Telefónica aún resulta posible imponer a los operadores de telecomunicaciones estrategias que se adaptan a una visión concreta del mercado, desechando otras alternativas que se imposibilitan5. Así, se dese cha la alternativa de favorecer la entrada de empresas que hacen uso de avances tecnológicos que podrían sal var lagunas de subdesarrollo. Por ejemplo, en 1997 British Telecom es descartada en las licitaciones, y en su lugar se favorece la entrada a operadores menos innovadores, como France Telecom y las empresas italianas, casos en los que la falta de competencia y los entornos oligopolistas en los que han sobrevivido les convierten en competidores menos amenazantes - y más comprensivos, si cabe, ante posibles compromisos con el monopolio español antes asentado6. La opción de marginar la entrada de empresas americanas cuenta no sólo con la bendición del partido de centro derecha en el gobierno, sino también con el apoyo del presidente del grupo de comunicación PRISA, que apoya al centro izquierda7. Por otro lado, la apuesta por las empresas italianas resultará fallida cuando estas inversiones se retiren de la península a finales de 2001.

Además, desde 1980 el panorama de excluidos y favorecidos es alterado de manera importante por la intervención de dos niveles de autoridad que antes no tenían protagonismo en el sector en el caso español, el nivel de la Unión Europea y el regional.

\section{El caso español a partir de 1980: cambios generales}

Los cambios que se producen en la década de 1980 vienen augurados por cambios políticos de gran magnitud, fruto sobre todo de la liberalización política con la transición de la dictadura a la democracia. Uno de los cambios significativos, que va a afectar a todas las áreas de políticas, es el cambio en los patrones de toma de decisiones (GUNTHER, 1996). D esde 1982 hay una tendencia al control directo de las políticas por parte del presidente del gobierno, que va acompañada de una reordenación de las áreas de políticas prioritarias. El papel del Parlamento pasa de la letargia durante el franquismo a nuevos patrones de participación parlamentaria, que posteriormente no se consolidan - ya que coincidiendo con el desarrollo de las autonomías y la entrada de España en la Comunidad Económica Europea, las Cortes se debilitan como arena pública de la política, a la par que se amplían los centros de decisión política (Q UINTANA, 1999: 420)8. También aparecen nuevos actores u organizaciones, que se asientan como instituciones, y que contribuyen a romper el monopolio del Ejecutivo y del Parlamento sobre áreas de políticas específicas?.

En lo referido a la implementación de programas concretos de desarrollo del sector de las telecomunicaciones en la sociedad, Antonio Alabau (1998: 41) encuentra resultados distintivos según el tipo de red de relaciones que se configura:

«Las variaciones son amplias, desde departamentos regionales o locales, institución o fundación que depende del gobierno regional, un departamento universitario, una cámara de comercio, una compañía de tecnología ligada a la región, una consultoría».

\section{La incorporación de nuevos actores}

Las instituciones políticas que cobran relevancia a partir de 1980 son los partidos políticos en el Parlamento, los tribunales, y un nuevo nivel de autoridad, el de las regiones. A estas nuevas instituciones, surgidas como fruto de la ampliación del espectro político, se le han de sumar los cambios que se producen en el ejecutivo como regulador, y la creación de instituciones, cuya inspiración está mas allá de España, en la UE.

Esla Constitución española de 1978 la que pone en marcha mecanismos potenciales para la incorporación de las nuevas instituciones - organizaciones, 0 actores políticos- en el sector de las telecomunicaciones

Para el caso de las Comunidades Autónomas, la Constitución legitima un sistema con un equilibrio muy sutil, que da lugar a focos de conflicto ausentes en un sistema federal como por ejemplo el estadounidense. La fragilidad del equilibrio viene dada porque en España la Constitución es muy ambigua, y las leyes de bases del gobierno central, como procedimiento establecido para desarrollarla, han sido muy específicas. Las leyes de ba- 
ses se han acercado en la práctica a los reglamentos - competencia a desarrollar por las Comunidades Autónomas- lo que a su vez les ha alejado de su intención primera, la de sentar bases ge nerales (0 LíAS, 1999). Por un lado, la indefinición constitucional no ha servido para acallar las pretensiones competenciales de gobierno central y autonomías. Por otro lado, la sobre-definición de las leyes de bases ha levantado heridas en las Comunidades Autónomas. El resultado práctico son niveles de conflicto crecientes entre las Autonomías y el Estado central, reflejados en los recursos de competencias y de amparo presentados ante el Tribunal Constitucional - que se incrementan a ritmo del 50 por 100 sobre cifras del año anterior en fechas recientes- 10.

A las nuevas esferas y niveles de autoridad, y a los focos potenciales de conflicto que subyacen en el espíritu y el desarrollo del texto constitucional, se suma la incorporación de España a la Unión Europea en 1987. Desde esta fecha existen nuevos canales a disposición de las Comunidades Autónomas para efectuar sus demandas, e incluso para intentar alterar el peso específico que tienen los distintos niveles de autoridad sobre las políticas (GIL, 2002). En las dos secciones siguientes se presentan los nuevos niveles y esferas de autoridad que vienen a reemplazar el monolítico y exclusivo nivel de autoridad del Estado central hasta 1980.

\section{Partidos políticos y regiones}

Durante el período de estudio se producen dos fenómenos inéditos en el panorama político español durante las cuatro dé cadas anteriores a 1970 (GIL, 2000: 79-185). Se crea un sistema de partidos, fruto de la liberalización política, que cambia durante la transición a la democracia. Los cambios se traducen en un reequilibrio de poder de los actores políticosil.

La actitud de los partidos políticos como actores respecto a la regulación de sectores económicos varía en los distintos Parlamentos y gobiernos elegidos. En las dos primeras elecciones democráticas, las dos Comunidades históricas del País Vasco y Cataluña cuentan con una representación importante en el Parlamento central como partidos políticos. El partido de centro Union de Centro D emocratico (UCD) no tiene mayoría suficiente para aprobar legislación y necesita su apoyo, pero el tema de la reestructuración sectorial no llega a la agenda política.

En las terceras elecciones generales de octubre de 1982, 202 escaños en el Parlamento, 26 por encima de la mayoría absoluta, le dan al partido de centro-izquierda (PSOE) las llaves del gobierno. Además de un nuevo gobierno, la elecciones marcan un cambio sustancial en el sistema de partidos característico durante la transición: «el partido de centro se desintegró, el partido comunista quedó reducido a la mínima expresión, y una fuerza conservadora alternativa emergió claramente a la derecha del espectro político» (PASTOR, 1996: 533).

En las elecciones legislativas de 1986, el Partido Socialista aún cuenta con mayoría absoluta, aunque pierde 18 escaños. En 1989 el PSO E logra aún una mayoría absoluta de los escaños, aunque más disminuida. H asta 1993 el Partido Socialista no se ve en la necesidad de recurrir al consenso con otros partidos políticos nacional istas 0 autonómicos para poder gobernar. D urante el período de la mayoría absoluta del PSO E, el papel de los partidos re gionalistas es más testimonial que efectivo. Ninguna mayoría puede vetar la legislación propuesta por el partido. Las elecciones de 1993 suponen una inflexión. Cuando el PSO E no tiene mayoría parlamentaria despierta agudamente el interés político por el sector de las telecomunicaciones, y se plantea la discusión sobre la alteración en la corriente de rentas existente. La necesidad de consenso entre partidos se convierte en imperativa cuando el proyecto de Ley de Telecomunicaciones por Cable es discutido en el Congreso12. Entonces CiU, partido nacionalista catalán, hace del compromiso sobre los contenidos del borrador el pilar básico, para seguir apoyando al gobierno minoritario del PSOE (SANZ y M arTín EZ-AlON SO, 1996: 28). Éste es un momento crítico, en el que los partidos políticos de representación autonómica mayoritaria comienzan a ser relevantes como actores políticos en la re gulación del sector de las telecomunicaciones, a la par que en otros sectores. El conflicto institucional que se plantea a propósito de la aprobación de la Ley de Telecomunicaciones por C able inaugura una nueva era de hacer política en España que se aleja de la mayoría absoluta parlamentaria y se acerca, durante el periodo del gobierno en minoría, al consocionalismo13.

\section{Tribunales}

Los tribunales se convierten en un nuevo mecanismo institucional útil para romper el privilegio del ejecutivo como ordenador del mercado de las telecomunicaciones bajo el marco regulador anterior. Este mecanismo institucional comienza a afirmarse con una sentencia del Tribunal Constitucional (TC) de 31 de enero de 199414. Por esta vía, el ejecutivo queda puntualmente incapacitado para limitar o prohibir la instalación de emisoras de televisión por cable en el ámbito local. Esta limitación supone el primer acotamiento de hecho del poder judicial al monopolio del ejecutivo como regulador. Como consecuencia de la limitación del TC, los ayuntamientos quedan capacitados para otorgar dos tipos de licencias; una, para el tendido de cables de telecomunicaciones en la vía pública dentro de los límites del munici- 
pio, y otra, para el uso de las redes de cable de telecomunicaciones destinadas a la transmisión de programas televisivos.

Además de una quiebra de la hegemonía reguladora del eje cutivo, esta sentencia es una señal de cambio para los empresarios. La señal indica que otros niveles de gobierno distintos al central pueden garantizar prerrogativas (GIL, 1996). Esto sirve de acicate extra para inversiones privadas de actores económicos antes excluidos en la partición de corrientes de rentas existente. Por otro lado, las diferencias entre ejecutivo y los tribunales restan interés a los grandes inversores enemigos del riesgo, ya que los derechos de propiedad no quedan bien delimitados. Las cifras de inversión de empresas de cable en estos años reflejan esta nueva confianza15.

La sentencia del Tribunal Constitucional es también un indicio de la necesidad de cambiar la política de protección al monopolio por parte del gobierno central16.

La sentencia del TC tiene otro efecto; al romperse de hecho el monopolio regulador del ejecutivo, aparecen los primeros conflictos entre dos niveles de autoridad, el gobierno central y los ayuntamientos. Uno de los primeros casos de desacuerdo es el del municipio de Barcelona, que lleva al enfrentamiento al alcalde Pasqual $M$ aragall (PSC -PSOE) y al M inistro José Borrell (PSOE), ambos del Partido Socialista (PSOE)17. A este contencioso entre gobiernos locales y gobierno central se une el Partido Popular (PP), opuesto al proyecto de Ley de Telecomunicaciones por C able avanzado por el Grupo Socialista. EI PP pasa de Popular a populista, y consigna a los alcaldes de su mismo signo político dar permisos de instalación a los empresarios dentro de los límites municipales.

La relevancia de la sentencia del Tribunal Constitucional va más allá del caso concreto de la televisión por cable. Representa el primer caso en el que un denunciante particular consigue romper la hegemonía del ejecutivo en materia de política de telecomunicaciones. Este precedente, habitual en el caso de los tribunales estadounidenses, es el primero de su signo en España para este sector concreto del mercado (GIL 2002: 22-24, 82).

Al papel del TC como esfera de autoridad autónoma se suman los tribunales de las Comunidades Autónomas. Estos tribunales pueden dictar sentencias apoyándose en la doctrina del Tribunal de Justicia Europeo en materias que reconozca el Tratado de la U nión. M ediante este recurso, los tribunales locales logran incluso desplazar la exclusiva del Tribunal Constitucional como intérprete de la Constitución18.

La sentencia de 31 de enero de 1994 del Tribunal Constitucional es una prueba fehaciente de que los tribunales pasan a ser una esfera de autoridad autónoma en los años 1990, sin sometimiento al ejecutivo. Como esfera autónoma de autoridad, los tribunales adquieren poder para mediar en la exclusión de actores en el mercado dentro del sector económico de las telecomunicacionesi19.

\section{Cambios institucionales y de políticas durante el periodo: el gobierno y el sector}

En la esfera de autoridad del ejecutivo se detectan cambios en la orientación de las políticas desde 1982, y comienza a producirse un cambio institucional importante con respecto al sector económico de las telecomunicaciones desde 1985. Los cambios de políticas responden a las dificultades planteadas por los desajustes macroeconómicos existentes en España a comienzos de los años 1980, y tienen como objetivo estabilizar la economía. Los cambios institucionales responden al desarrollo de la capacidad autónoma de gobierno del ejecutivo respecto de los intereses regulados.

En un principio, el cambio de sistema político que se produce con la transición de la dictadura a la democracia tarda en tener un impacto drástico en sectores del mercado como el de las telecomunicaciones. En los primeros gobiernos de la transición, cuando este sector recibe atención política es como resultado del compromiso del gobierno con las políticas de estabilización económica20 Como consecuencia del programa de reforma de la economía se aplican las políticas monetarias necesarias para alcanzar la estabilización, pero no se abordan reformas de la empresa con participación estatal21.

Las primeras señales del cambio de políticas se detectan en cambios de pautas establecidas, según las cuales el monopolio telefónico diseñaba su propia estrategia de expansión y de integración vertical con otras empresas subsidiarias22. A partir de 1982, el primer gobierno socialista cuestiona las prácticas del monopolio, en el que el Estado posee una participación importante, pero no mayoritaria23. En febrero de 1982, seis meses antes de ganar las elecciones, el grupo socialista presenta una proposición de ley para queTelefónica transfiera al Instituto $\mathrm{N}$ acional de Industria su holding industrial24. En 1985 el monopolio se desmarca de las empresas subsidiarias dejando parte del grupo en manos de la empresa AM PER. El cambio parece inspirado por el M inisterio deEconomía, que se opone a la política industrial de Telefónica y del M inisterio de Industria (GAM ELLA, 1991), y refleja la nueva filosofía de los gestores para reducir costes. En este momento los cambios de estrategia frente al monopolio son en gran parte el re sultado de la crisis económica. Crisis heredada por los socialistas en 1982 despues de la segunda crisis del petróleo, que arruina parte de los resultados de los Pactos de la M oncloa, dejando como herencia unas tasas de crecimiento de -0,1 por 100,-1,2 por 100 y -0,2 por 100 en 1979, 1980 y 1981, respectivamente (M ARAVALL, 1996: 467). A la crisis económica se une la experiencia truncada de expansión económica de los socialistas en Francia 
en 1981 y ante esta experiencia de resultados negativos, el gobierno socialista en España opta por el ajuste económico (PASTOR, 1996: 537). El cambio de orientación drástico hacia el monopolio responde en primer lugar a este objetivo gubernamental.

La participación accionarial del Estado en el monopolio sirve en este caso de caballo de Troya para forzar cambios como el cese del director general del área de planificación del monopolio25, y el estancamiento de la política industrial. A su vez, el gobierno abandona el seguimiento de las condiciones de aportación tecnológica previstas de empresas extranjeras beneficiadas con magras ayudas públicas para su localización en España (GAm ELLA et al., 1991). Todo esto ocurre a la vez que desde el M inisterio de Economía y H acienda se imponen restricciones a la inversión de Telefónica. Como alternativa, la empresa negocia con el gobierno subidas en los precios de las tarifas para conseguir autofinanciarse 26 - subidas siempre difíciles dado que los gobiernos pre fieren tarifas bajas para contener la inflación- .

Con la desvinculación del monopolio de su estrategia industrial no desaparece la protección prestada por el Estado frente a competidores potenciales, si bien el cambio de políticas tiene un objetivo claro en la rentabilidad, mediante el cambio de estrate gias del monopolio. En primer lugar, se aprueban emisiones de acciones en las bolsas internacionales27. En 1989 la participación extranjera se eleva ya al tope del 25 por 100 previsto por la ley28. En segundo lugar, el monopolio se concentra en la operación de la red pública de telecomunicaciones, sobre la cual tiene derechos exclusivos29. Además, la compañía comienza su expansión internacional, con proyectos de participación y explotación de redes, en países de América Latina principalmente30. Este cambio de estrategia cuenta con la bendición y el apoyo tácito del ejecutivo.

H asta 1985 se producen los cambios de políticas más importantes inspirados por el ejecutivo sobre el sector. D esde esta misma fecha se producen cambios institucionales en el seno del ejecutivo mismo. Desarrollando capacidad de gobierno sobre el sector de las telecomunicaciones, el ejecutivo inspira la creación de la Dirección General de Telecomunicaciones y la Secretaría General de Comunicaciones en 198531. Estas instituciones se crean dentro del M inisterio de Comunicaciones para coordinar a las que existían previamente con competencias sobre la mate ria32. Los poderes de ambas son sobre todo formales, hasta que se aprueba en 1987 la Ley de 0 rdenación de las Telecomunicaciones de 18 de diciembre (LOT), y se firma un nuevo contrato del Estado con Telefónica en 1991. Ambas instituciones fueron encargadas de la redacción de la LOT, «la primera regulación comprehensiva del sector de las telecomunicaciones en España» (JORDANA, 1997: 16). Muestra de la importancia de ambas agencias es el crecimiento en el número de funcionarios, que pasa de 40 en 1985 a 840 diez años más tarde33.
D esde 1987 la LOT es inspiradora de cambios institucionales que no sólo afectan al ejecutivo regulador. Por esta ley, y como resultado de las presiones para participar de otros sectores, se crea un Consejo Asesor de Telecomunicaciones (CAT ), que es un espacio nuevo de participación34. La primera función del CAT es ser notificado de las medidas que trate de poner en práctica el gobierno concernientes a las telecomunicaciones. En su composición se incluyen representantes de distintos niveles de gobierno, sectoriales, sindicatos, usuarios y profesiones reguladas. Un impacto importante de la puesta en marcha del CAT es la mejora del problema de información asimétrica, gracias a la creación de nuevos canales para su difusión. La institucionalización del CAT contribuye al aumento de la transparencia sobre los grupos económicos y sociales con intereses en el sector.

En 1993 se producen nuevos cambios de políticas e institucionales. Estos cambios se adoptan cuando el ejecutivo comienza a sensibilizarse sobre la necesidad de trasponer la normativa comunitaria sobre el sector al ordenamiento jurídico español, y también debido a presiones internas35. Tales cambios afectan a la posición que ocupa el gobierno respecto de la industria y de otros niveles y esferas de autoridad. En este momento el Partido Socialista cuenta con un gobierno en minoría, lo que le obliga a pactar con otros actores políticos antes excluidos del marco regulador del sector - como los partidos políticos nacionalistas, que en el caso de CiU y PN V ya gobernaban en sus Comunidades Autónomas desde 1980, y los gobiernos de las propias Comunidades Autónomas- . Por lo tanto, los cambios tienen la impronta de la participación de niveles de autoridad antes ausentes de la regulación del sector.

A pesar de los cambios mencionados, se puede concluir que en esta etapa no hay cambios drásticos en la forma de exclusión practicada en el sector de la telecomunicaciones ${ }^{36}$. JORDANA (1999: 235) señala como característica la lentitud del «proceso de diferenciación entre la emergente administración especializada en telecomunicaciones y el operador entonces monopolístico.» Este proceso se desarrolla intentando no afectar a los intereses de Telefónica, «empresa a la que el gobierno consideraba como su campeón nacional en la competición global de las telecomunicaciones que ya se perfilaba» (JORDANA 1999: 215).

En una primera etapa, la Ley de 0 rdenación de las Telecomunicaciones, que se desarrolla por el Real Decreto 1066/1989, reserva:

«a Telefónica la prestación en régimen de monopolio de todos los servicios de telecomunicaciones, ya que incluso transitoriamente clasificaba como servicios finales o portadores reservados al Estado al gunos que eran de valor añadido hasta la adopción de los correspondientes reglamentos técnicos de regulación de servicios concretos (como fue el caso de los servicios de radio búsqueda y gestión de redes), y esa in- 
sostenibilidad [de la reserva] se agudizó a la vista de las tendencias liberalizadoras de la Unión Europea» (ARPÓN DE MendíviL, 1995: 2).

Cuando en 1992 el gobierno lleva a cabo cambios en la LOT de 1987 para trasponer la directiva 90/388/EEC 37 el tema central de desarrollo de la directiva europea no queda zanjado. El nivel de autoridad del Estado central continúa protegiendo a Te lefónica a juzgar por la reacción a nivel comunitario. La LOT define tel efonía básica en el párrafo 15 de su anexo coincidiendo con los términos en los que lo hace la directiva. Sin embargo, y a pesar de las quejas reiteradas de la Comisión,

«parece que la interpretación de las autoridades españolas de esta definición no ha sido clara y que, aunque definida en la ley, una orden administrativa especial sería necesaria para definir más claramente el monopolio de la telefonía básica de Telefónica. Esta definición [en el año 1995] aún no ha sido adoptada.» COM (95) 113 final, p. 40.

La LOT es sustituida por la Ley General de Telecomunicaciones, de 2 de abril de 1998 - «a pesar de que el compromiso contraído por España con la Comisión obligaba a aprobarla antes de finalizar 1997»(SANZ 1999: 122)38-.

Esta protección en forma de garantías específicas y que sirven para excluir a posibles competidores se observa también en el Acuerdo sobre la Política de Telecomunicaciones del período 1994-1998 aprobado por el nivel de autoridad del Estado central39. A esto se añade en la práctica que las condiciones para otorgar licencias en al gunos servicios designados como de valor añadido a la telefonía básica son más restringidas que aquellas contempladas por las directivas comunitarias40. La definición amplia de los servicios de valor añadido le permite al nivel de autoridad del Estado central mantener un ámbito de restricciones a posibles competidores. El proyecto de Ley de Telecomunicaciones por C able de 1995 es criticado también por sus mecanismos de retraso en la incorporación de nuevos actores a al gunos segmentos del mercad041. El retraso en la incorporación de competidores también ha afectado a nuevas inversiones nacionales extranjeras con la moratoria negociada por el gobierno español para abrir el mercado de telecomunicaciones sólo a partir de 199842.

La política de telecomunicaciones del gobierno de centro de recha del Partido Popular ha sido tachada de continuista en sus líneas generales respecto de aquella puesta en marcha por el ejecutivo social ista hasta 1995. Aunque no es el objeto de este estudio resaltar las diferencia entre ambas, una diferencia de tipo cualitativo respecto del período del gobierno socialista y anteriores es el interés del ejecutivo desde 1999 por la «Sociedad de la Información» (SI )43, que puede plasmarse en un cambio en ciertos mecanismos de exclusión. Este interés contrasta con la observación de JORDANA para el periodo precedente: «fue sorprendente, a medida que pasaban los años, el escaso interés mostrado por el gobierno central por establecer una política propia respecto a los temas relacionados con la promoción y difusión de la SI, incluyendo el establecimiento de plataformas de coordinación eficaces entre las distintas administraciones públicas y los agentes sociales» (1999b: 237).

La forma en que se plantea ese interés por la SI puede cambiar el equilibrio de esferas de autoridad para el sector al nivel del Estado central. A parte de la creación de una comisión interministerial de ciencia y tecnología el 27 de Julio de 1999, el gobierno de centro derecha comienza poniendo en manos del Ministerio de Industriz44 el impulso de la SI - definida como el uso de las telecomunicaciones, Internet y los ordenadores en general- . Tras las elecciones ge nerales de 12 de marzo de 2000, el PP logra una mayoría absoluta en las urnas. El gobierno conservador reparte entonces las compe tencias sobre telecomunicaciones entre los M inisterios de Ciencia y Tecnología (M CyT) y la Comisión del M ercado de las Telecomunicaciones (CM T). En total, desde este momento y hasta la actualidad, son varias las agencias y $M$ inisterios implicados en al gún aspecto de la regulación de las telecomunicaciones (el M CyT, la CM T, el M inisterio de Economía - bajo cuyo paraguas institucional sesitúa el Tribunal de D efensa de la Competencia- la Comisión Interministerial que fija los precios finales - Comisión D elegada del Gobierno para Asuntos Económicos- y el M inisterio de Hacienda, encargado de negociar y cobrar la tasa radioeléctrica. Esta variedad en la práctica induce a confusión y genera críticas por parte de los actores económicos interesados en el sector.

Referente a las medidas reguladoras en el ámbito del fomento de la competencia, desde el M CyT, se impulsa la competencia en llamadas locales y la liberalización del bucle local (Tomé 2001: 7)45. Por su parte, la CM T interviene ex ante spara ordenar lo que debe hacerse mientras se consigue una nueva situación de competencia» (VÁzQuez QuintanA, 2001:3 2).

En cuanto a aquellas empresas que habían estado integradas verticalmente al monopolio en los años 1970s y 1980s, existen entre ellas lazos de cooperación. La desvertebración del modelo de integración vertical no es sustituida por relaciones de acomodo de la oferta y la demanda bajo un modelo de exclusión por medio de la competencia si no que sigue siendo el Estado el garante del tipo de exclusión. A nivel empresarial existe colaboración en asociaciones como CIECAT (Círculo Español para la Calidad en Telecomunicaciones) 46. La pauta de formación de la asociación es un núcleo de empresas muy representativas del sector de las telecomunicaciones como Alcatel, Standard Eléctrica, Ericsson, Lucent Technologies, Amper, Telefónica de España y Telefónica Investigación y D esarrollo. D esde este núcleo se busca atraer e incorporar a otros sectores y empresas. En la estructura de la asociación tienen prioridad los «miembros de pleno derecho», que están facultados para participar en todos los órganos de la asociación. Los «miembros asociados» son empresas medianas 
y pequeñas cuya actividad principal no es la electrónica, la informática o las telecomunicaciones. Los miembros asociados pueden participar en los Comités Tecnológicos pero están excluidos de los órganos de gobierno. Por último, el grupo de los «colaboradores» compuesto por representantes de universidades y organismos públicos de investigación, que también participan, a título personal, en los Comités Tecnológicos (Ad elL y CaVero, 1997: 99).

\section{Instituciones y mecanismos de inspiración comunitaria}

A partir de la entrada de España en la Comunidad Europea en 1986 se da un contenido diferente a instituciones ya existentes y se adoptan otras nuevas. Estas instituciones ejercen efecto como esferas autónomas de autoridad para la regulación del sector de las telecomunicaciones. Para el caso español son importantes el Tribunal de D efensa de la Competencia (desde 1989) y la Comisión del M ercado de las Telecomunicaciones (creada en 1997).

El estudio de estas esferas de autoridad encuentra la dificultad del corto plazo en el que estas instituciones han tenido impacto autónomo y distinto al del ejecutivo sobre el sector. El caso de Estados U nidos es un ejemplo de cómo las instituciones pueden llegar a desempeñar funciones no vislumbradas en su creación (GIL, 2002: 1-45). Por esta razón el análisis de las instituciones españolas que se desarrollan en la década de los años 1990 es un compromiso entre la descripción de sus estatutos, la discusión sobre los efectos de sus resoluciones y de las relaciones que se perfilan entre ellas en poco más de un lustro47. Tal análisis no puede ser categórico sobre los efectos que se derivarán de la interacción entre unas instituciones que sólo comienza a perfilarse.

La pregunta pendiente es si instituciones creadas claramente con la intención de cambiar parcialmente el tipo de exclusión dominante en el mercado serán en algún momento plataforma garante de futuros cambios para la incorporación de grupos económicos diferentes - como se observa en el caso estadounidense (GIL, 2002: 41) - o bien su efecto se agota con el cambio de políticas que favorece un tipo de competencia garantizada por el Estado.

\section{El Tribunal de la Competencia}

Dos instituciones dependientes del M inisterio de Economía se encargan de la aplicación de la Ley de Competencia, el Tri- bunal de D efensa de la Competencia48 y el Servicio de D efensa de la Competencia. El Tribunal de D efensa de la Competencia ha tenido un papel relevante en la ruptura del consenso monolítico del gobierno sobre telecomunicaciones49. A pesar de su nombre, el Tribunal no forma parte del poder judicial, sino que se crea como una agencia especializada dentro del M inisterio de Comercio.

En un primer momento, el Tribunal es una institución vacía de efectos, no sirve para introducir liberalización en el mercado ni para inducir la retirada del Estado de la economía. Como institución, el Tribunal no consta con mecanismos adecuados para llevar a cabo su cometido50. La falta de mecanismos para que el Tribunal ejerza las funciones para las que se crea se subsana desde 1989, tres años después de la entrada de España en la Comunidad Europea, ya que con esta fecha el Parlamento aprueba una nueva Ley de D efensa de la Competencia, inspirada en la ley comunitaria, y modificada parcialmente en diciembre de 1999 y febrero de 200251.

La posición del Tribunal es destacada en la práctica posterior dentro del panorama nacional gracias a sus relaciones con la D G IV dela Comisión Europea52.

Existe un mecanismo que introduce la posibilidad de contestación de las decisiones del Tribunal por parte de particulares, éstas pueden ser apeladas a la Audiencia N acional, y después al Tribunal Supremo.

Un mecanismo adicional que funciona como canal de ape lación de nuevos intereses es el Servicio de Defensa de la Competencia (SDC)53. EI SDC puede tomar la iniciativa en la iniciación de investigaciones sobre sectores económicos 0 puede ser requerido a hacerlo por cualquier parte interesada. Tras el examen de asuntos relacionados con el D erecho de la competencia, el SDC decide elevarlo o no al TDC54. Esta institución se encarga también del Registro de D efensa de la Competencia55.

La influencia reguladora del Tribunal comienza a ser patente con la publicación de un informe y dos decisiones contra la posición de dominio del monopolio en 1993 y 199556.

El informe del Tribunal publicado en 1993 se decanta ya por un cambio en el tipo de exclusión, para lo cual aporta razones de eficiencia57. Se recomienda el incremento de la competencia en el sector servicios, entre los que se incluyen las telecomunicaciones. Estas recomendaciones han sido seguidas de condenas de posición dominante en fechas posteriores 58.

A pesar de no representar cambios drásticos en el tipo de exclusión, el valor de las decisiones y el informe estriba en apuntalar la autonomía del ejecutivo visa visel monopolio. 


\section{LaComisión del M ercado de las Telecomunicaciones}

En el año 1997 se crea una nueva institución para regular el sector de las telecomunicaciones, la Comisión del M ercado de las Telecomunicaciones (CM T) 59. Cuando esta institución se instaura, se espera que responda al objetivo de velar por la competencia en el sector, así como a criterios de mayor independencia política.

Las asignaciones de competencias muestran el compromiso del gobierno central con una apertura a la competencia con garantías para las empresas. G arantías tanto para inversiones posibles como para la creación de nuevos derechos de propiedad garantizados por el Estado. El garante de la competencia sigue siendo el Estado mismo. Por esta razón, puede hablarse de un cambio sólo parcial en el modo de exclusión dominante. Al tipo de competencia que trata de instaurarse no es en ningún modo ajeno el Estado.

En cuanto a los problemas de tipo práctico para cumplir con las misiones encomendadas, en el caso español, al comienzo del funcionamiento de la CM T se plantean problemas para distinguir las tareas entre el Tribunal de D efensa de la Competencia y la CM T 60. Un segundo problema, que no sólo afecta a la función de velar por la mayor competencia, sino también a la capacidad de independencia respecto del ejecutivo es que con la simple función de informe al gobierno, la CM T ya se ve desbordada61. Estos problemas no invalidan a la CM T como referente, aunque incluso tras las elecciones generales de 12 de marzo de 2000 continúan recayendo en $M$ inisterios dependientes del ejecutivo importantes decisiones que afectan a la asignación de corrientes de rentas existentes, como el M inisterio de Fomento (antes de 2000)62, el M inisterio de Ciencia y Tecnología, el Ministerio de Economía, la Comisión Interministerial que fija los precios finales (Comisión D elegada del Gobierno para Asuntos Económicos), y el M inisterio de $\mathrm{H}$ acienda - encargado de recaudar la tasa radioeléctrica- . Esto significa que muchas decisiones continúan siendo dependientes del ejecutivo.

Respecto a la medida de la independencia política de la CM T, al hablar de independencia política de una comisión es necesario precisar lo que se entiende por tal independencia63. En los estudios comparados se ha traducido, bien como independencia del Parlamento, bien como una mayor diferenciación respecto del ejecutivo, o bien como una mayor especialización. El calificativo de «nndependiente» a agencias o comisiones resulta banal a menos que se precise independiente de que otras esferas y niveles de autoridad y cómo. Ilustrativos en este sentido son los tipos de dependencia política estatutaria de comisio- nes reguladoras del mercado sectorial de las telecomunicaciones en Estados U nidos y Gran Bretaña. En los Estados Unidos la comisión es una creación del Congreso, en quien recae constitucionalmente la autoridad reguladora de sectores económicos con intercambios entre Estados federados. El Congreso crea la agencia reguladora (FCC) y puede abolirla. Puede reducir sus recursos restringiendo su presupuesto o nombrando a miembros poco competentes. Estatutariamente también el ejecutivo tiene ciertos poderes para efectuar nombramientos. Estatutariamente, pues, y en la práctica, la agencia es dependiente del eje cutivo y el legislativo.

En España, a nivel de medios de comunicación y entre los mismos miembros de la Comisión, existe una idealización de OFTEL (O ffice of Telecommunications), la agencia reguladora británica, como órgano independiente al que emular64. En el caso de Gran Bretaña, la Comisión independiente no es Comisión, si no un departamento (OFTEL) encabezado por un Director G eneral dependiente de una cartera ministerial, parte a su vez del ejecutivo65. Todos los poderes legales recaen en el Director General, un solo individuo66. El modelo de agencia reguladora instaurado se caracteriza por un rechazo del modelo de la comisión porque existe el recuerdo de comisiones reguladoras en el siglo XIX excesivamente legalistas (Prosser, 1997: 45). En el pasado, el exceso de legalismo se convierte en un problema, entre otras razones porque los costes de los litigios son prohibitivos (Prosser, 1997: 36). Un análisis dinámico de $0 \mathrm{FTEL}$, la agencia reguladora británica pone de manifiesto que la institución sufre cambios progresiVOS (HULSINK , 1996: 194)67. En un principio, OFTEL recibe numerosas críticas por su falta de transparencia (en contraste con la Comisión FCC de los Estados Unidos)68. En años sucesivos se han puesto en marcha reglas formales e informales que funcionan como mecanismos para poner coto al poder de la agencia respecto de otros intereses políticos, económicos y sociales, además de la falta de transparencia. En este sentido, los tribunales británicos han jugado un papel importante al solicitar que las decisiones de OFTEL estén fundamentadas. Este tipo de relaciones entre esferas de autoridad no se contempla estatuariamente en un principio, son el resultado de la práctica política posterior.

En el caso español, según los estatutos, la Comisión reguladora (CM T) ha variado en su composición entre los ocho y los nueve miembros, aunque comenzó funcionando con siete. En el nombramiento de sus componentes, que se realiza por el gobierno, ha existido influencia de formaciones políticas con representación en el Parlamento central debido a que el partido en el gobierno no goza de mayoría parlamentaria entre 1996 y 199969. Así, llegan a ser hasta siete los partidos con influencia para proponer al M inisterio al menos un miembro de la cúpula del regulador70. 


\section{Conclusiones}

Como conclusiones del período 1980-2002 puede destacarse en primer lugar una constante: cuando nos centramos en el eje cutivo como regulador único, se producen cambios de políticas y cambios institucionales. Sin embargo, desde el nivel de autoridad del Estado central, no se produce un cambio en el modo de exclusión practicado en el mercado mientras éste concentra el poder regulador exclusivamente en el ejecutivo. Cuando el ejecutivo tiene la exclusiva reguladora se ponen en marcha cambios en las estrategias del monopolio, así como cambios institucionales, cuyo fin no parece ser el que se produzca un cambio drástico en el tipo de exclusión, sino un cambio en los objetivos de las políticas económicas de la compañía y el gobierno mismo. Como se ha visto, ni siquiera la desintegración de la integración vertical practicada por Telefónica se traduce en la incorporación de nuevos competidores principalmente, sino en el cambio del tipo de colaboración entre las empresas711. D esde esta óptica, es posible concebir casos de sectores económicos en los que habiendo un matrimonio de hecho entre el regulador y la compañía regulada, se producen cambios en las políticas, incluso cambios institucionales. Estos cambios pueden tener el objeto de avanzar fines distintos, como una mayor eficiencia o el cambio de estrategias comerciales, sin por ello alterar el tipo de exclusión predominante en el sector. De modo que refiriéndose al esquema planteado en la sección teórica, el cambio institucional es una condición necesaria, pero no suficiente, para lograr cambios en el modo de exclusión en el caso español. D ependerá en todo caso de si se incorporan o no nuevos actores con reivindicaciones diferentes sobre la partición de la corriente de rentas existente.

En el caso español, el gobierno se arma con capacidad institucional gradualmente, ya que le resulta trabaj oso diferenciar entre sus intereses y los de la compañía, y aun más llevar esta diferenciación a la práctica. Sin embargo, durante esta etapa (1985-93) el ejecutivo consigue desarrollar cierta capacidad de autonomía visà visel monopolio. Mientras el ejecutivo es el único regulador, que además garantiza el patrón de exclusión predominante sobre una sola compañía y tiene participaciones en la empresa misma, resulta difícil dibujar una línea clara de separación entre intereses gubernamentales e intereses de otros grupos sociales representados en la empresa, éstos tienden a coincidir en ausencia de arreglos institucionales que facilitan esta diferenciación. Es más, en el caso español se crean inercias y mecanismos que protegen la asociación entre ambos, y que por lo tanto favorecen la falta de incorporación de nuevos actores económicos y sociales. Éstos incluyen la vía de las innovaciones tecnológicas, dado que el tipo de contrato de Telefónica con el Estado permite decidir sobre la rapidez de la incorporación o no de las innovaciones, y pone a disposición de la empresa el control de un me canismo clave para imposibilitar el cambio de corrientes de rentas, el veto a la incorporación de nuevos actores que podría derivarse de los adelantos tecnológicos.

D ebido a la falta de adaptabilidad institucional para que se cuestionen y cambien corrientes de rentas en España hasta la dé cada de 1980, las expectativas se centran en la puesta en marcha de una serie de políticas paralelas, cuyo fin es cambiar las pautas de comportamiento de los actores involucrados, mediante el cambio de las instituciones mismas. En el período estudiado se observa que el cambio en el tipo de exclusión se produce parejo y como consecuencia de modificaciones en las instituciones re guladoras. Algunas son de nueva creación, como las agencias especializadas, otras como los tribunales estaban latentes, pero no habían jugado antes un papel como esfera de autoridad autónoma en el sector de las telecomunicaciones.

En el ámbito de la polity el cambio tiene tres vertientes, la primera es un redimensionamiento del Estado central. Se fomenta el fortalecimiento, siempre relativo, de la autonomía del Estado respecto del actor con rentas de monopolio desde la dé cada de los 1920 hasta finales de la década de 1980. Este fortale cimiento se produce en dos fases desde 1982. En la primera, el M inisterio de Economía y el de Industria se desvinculan de la política industrial del actor monopolístico y de los subsidios a sus proveedores. En la segunda, se crean instituciones con un poder de información autónomo respecto del actor monopolísti$\mathrm{CO}^{72}$. Junto a un nivel estatal central de nuevo cuño, destaca el papel de los tribunales, que comienzan a cuestionar las políticas anteriores, protectoras de rentas de monopolio. De manera similar a la de los tribunales estadounidenses, emiten sentencias que tomadas separadamente son discretamente visibles desde 1985. Sin embargo, la suma de tales sentencias representa una afirmación de la diferencia entre esferas de autoridad - ejecutivo, tribunales- así como cambios cualitativos de criterio sobre el papel del Estado como garante de rentas de monopolio. Se observa una diversificación de esferas de autoridad con peso específico para influir en el sector, mientras que, anteriormente, la esfera de autoridad por antonomasia era el ejecutivo, que monopolizaba también el rol de único nivel de autoridad.

La segunda vertiente del cambio institucional, y la más poderosa en el corto plazo, es la aplicación doméstica de directivas de la Unión Europea que cuestionan frontalmente los flujos de rentas existentes hasta comienzos de los años 1990. Como consecuencia de esta europeización se promulga una nueva Ley de la Competencia en 1989, comienzan a trasponerse las directivas europeas desde 1992 y se crea una agencia como la Comisión del M ercado de las Telecomunicaciones en 1997.

Sin embargo, la investigación empírica muestra que la realidad española no es el resultado único de la aplicación de normas legales provenientes del ámbito de la Unión Europea, en cuyo 
espacio existen variaciones en el tiempo y en el modo de implementación de directivas entre países, y reivindicaciones reguladoras de nuevos niveles y esferas de autoridad a nivel estatal.

La tercera vertiente del cambio institucional se sitúa en el nivel regional, que no se aborda en este trabajo. Algunos gobiernos de las Comunidades Autónomas promocionan proyectos de telecomunicaciones regionales, incluso se convierten en actores económicos en el sector. Estos gobiernos regionales llegan a actuar como estructuradores de intereses sociales y económicos en sus espacios territoriales, con reivindicaciones propias sobre las rentas en el sector de las telecomunicaciones. El hecho de que ni la Constitución de 1978, ni el desarrollo de los Estatutos de Autonomía, zanjen la cuestión de los límites a las reivindicaciones de las Comunidades Autónomas, refleja también el grado de incertidumbre institucional sobre el poder efectivo de los niveles de autoridad para regular actores económicos en el caso español.

En España, a la luz del modelo explicativo y de las hipótesis que tratan de probarse, las expectativas de cambio de políticas de los actores no están puestas en el uso flexible de unas instituciones consensuadas; el cambio en las pautas de comportamiento pasa por la expectativa de inducir cambios institucionales. Esto es consecuencia de la tradición existente; un marco institucional resistente a los cambios por medio de la incorporación de nuevos grupos sociales y económicos a la corriente de rentas protegida hasta la década de 1980. Tal marco institucional modela las expectativas de los actores - sociales, políticos, económicosde manera que para cambiar las pautas de comportamiento, éstos estiman mas créble el cambio del marco institucional. El cambio del marco institucional, sin embargo, tiene límites definidos que no coinciden exactamente con un modelo del mercado en el que predomine la lógica de la competencia, ya que por un lado el Estado tiene prerrogativas importantes a la hora de decidir que actores se incorporan como competidores. Además, resulta de interés para las empresas ya establecidas que las garantías estatales que excluyen a competidores no desaparezcan del todo. Esto es consistente con el deseo de grandes empresas multinacionales de que la UE se implique en programas de infraestructuras, así como de investigación y desarrollo - y se olvide de temas de regulación de derechos sociales o de compromiso social - . Para el Estado central como nivel de gobierno la desregulación también tiene el limite de no socavar sus propios intereses mediante la erosión de la base fiscal sobre un sector dinámico como el de las telecomunicaciones 73 .

\section{Notas}

* Dra. por el Instituto Europeo de Florencia. ogil@ceacs.march.es. Investigadora visitante del CEACS. Este artículo tiene su origen en un capítulo del manuscrito «Las Telecomunicaciones en Estados Unidos y España (1875-2002)» M adrid: CIS, 2002. La autora quiere agradecer el valioso apoyo institucional del CEACS (Fundación Juan $M$ arch) en el trabajo necesario para la revisión de esta investigación.

1 El País, 11 de enero 1997, p. 39.

2 Un ejemplo es el de los tribunales en Estados Unidos, y su papel básico en temas anti-monopolio, que ha llevado a alteraciones puntuales e incluso drásticas del tipo de mercado a lo largo de este siglo.

3 Estudios recientes de economía política ponen de relieve que los sectores económi$\cos$, que apoyan a partidos políticos, tienen altos incentivos para mantener el apoyo de forma constante al mismo partido (M CEwON, 1999, VerDIER, varios años).

4 En ese momento, Airtel es un consorcio liderado por los Bancos Santander y BCH $(13,7$ por 100 cada uno), cuenta con socios estadounidenses (Airtouch, 15,7 por 100) y británicos (British Telecom, 6,31 por 100). Participan tambien las cinco cajas de ahorro (16,8 por 100), Ineuropa (10,5 por 100), Unión Fenosa (7,8 por 100) y la Corporación Alba (2,63 por 100). El capital español suma el 72,9 por 100 (El País, 4 de octubre de 1995, p. 62).

5 «C ompetencia en cuarentena: el gobierno ha provisto a Telefónica de las armas suficientes para contener a Retevisión a largo plazo» (El País, 20 de julio de 1997, negocios, p. 2)

6 El artículo de M ONTERO (1999) «Salvaguardia de la competencia o de los competidores?» ofrece junto con un análisis de los mecanismos de exclusión, el listado exhaustivo de los favorecidos por la prerrogativa estatal de un marco «favorecedor de la competencia».

7 Jesús de Polanco, quien a propósito de la entrada de USW EST en C atal uña decla ra: «Se ha metido un norteamericano en España por una puerta falsa [...] Esa unión del Ayuntamiento de Barcelona y US W est es uno de los disparates políticos más sorprendentes.[...] Los norteamericanos llegan para operar en telefonía y no precisamente para ofrecer películas, y se han colado por una puerta falsa» (El País, 28 de septiembre de 1995, p. 32).
8 U sando la distinción de 0 STROM , entre tres tipos de reglas, las constitucionales, las reglas Ilamadas reglas colectivas de segundo rango, y las reglas operacionales, lo ocurrido a partir de 1980 en España es que actores de ámbitos sectoriales logran extricar del Parlamento el monopolio del debate sobre las reglas operacionales, y en ocasiones, también sobre reglas colectivas de segundo rango. Estos actores u organizaciones negocian estas reglas mencionadas en instituciones políticas mas restringidas y mas aisladas de los vaivenes electorales. Una muestra de este fenómeno la ofrece el sector de las telecomunicaciones durante gran partedel período. Una excepción en este sentido es la Ley de Telecomunicaciones por Cable, que se convierte en un debate prioritario en el Parlamento del gobierno central en 1995. En 1999 los Parlamentos de dos Autonomías con televisiones propias, el País Vasco y Cataluña, cobran una importancia antes desconocida en el nombramiento de los comités directivos de estas televisiones - que antes se reservaron siempre los ejecutivos autonómicos- . Sin embargo, estas excepciones pueden haber sido más bien coyunturales.

9 En este último sentido, a nivel de la política del Estado central, y en lo referente a la renegociación de las rentas de monopolio en el sector de las telecomunicaciones, la «existencia de arenas de negociación sectorial entre el gobierno y los actores socio-políticos refuerza la posición marginal y periférica de los grupos parlamentarios en relación con los circuitos de decisión. Asimismo, los órganos superiores de la Administración (secretarios de Estado, subsecretarios, secretarios generales, directores generales, gabinetes ministeriales) intervienen y desempeñan un papel relevante en el proceso de selección y filtro de las decisiones a tomar relativas a la actuación e intervención de los poderes públicos» (Q UINTANA, 1999: 420-421).

10 En la presentación de la M emoria del Tribunal Constitucional de 2000, el presidente de la institución, Pedro Cruz Villalón, se queja del aumento de asuntos quellegan a la institución, «en especial en conflictos de competencias entre el Estado y las Comunidades Autónomas, que en 2000 crecieron en un 50 por 100 sobre el año anterior, así como 6.762 recursos de amparo, más de 1.200 por encima de 1999 》(El País, 14 de junio de 2001, p. 15).

11 El cambio en el sistema de partidos obedece a la distinta representación parlamentaria de los mismos en diferentes períodos. Por un lado, desaparece el gran aglutina- 
dor que había sido el partido de centro, Unión de Centro D emocrático (UCD), en los primeros años de la transición. Por otro lado, el sistema oscila entre la mayoría parlamentaria del partido de centro izquierda (PSOE), y la del centro derecha (PP), pasando por un periodo (1996-1999), en el que gran cantidad de votos premian a dos partidos nacionalistas, CiU en Cataluña (partido septuagenario) y PN V en el Pais Vasco (partido casi centenario).

12 Sobre la economía política de la regulación de las comunicaciones por cable en España véase G IL (1996), y sobre el conflicto institucional que se plantea véase SANZ (1996).

13 GIL (1995): «0 n W hether or nota critical juncture is taking place in Spanish politics. The Structuration of an Economic Sector and $\mathrm{H}$ ow the Roles of the Central and the Regional Governments are being Redefined» Propuesta de investigación empírica premiada bajo el programa Salvador de M adariaga del gobierno español en el Instituto Universitario Europeo, Florencia. Si bien, como indica Przew ORSKI: «se debe te ner en cuenta que las mayorías electorales han sido raras en la historia de las democracias exitosas; en el periodo de postguerra, solo una elección de cada quince ha resultado en una mayoría de votos para un solo partido. D e modo que la mayoría de las democracias son gobernadas, bien por coaliciones explícitas entre partidos, ninguno de los cuales puede gobernar por sí mismo, o por gobiernos en minoría, basados en un apoyo implícito asegurado» (1991: 36).

14 El Tribunal Constitucional (TC) se pronuncia afirmando que, al no existir una ley sobre la materia, se ha de respetar la creación de nuevas emisoras de Televisión por Cable, basándose en los principios de libertad de expresión e información recogidos en la Constitución. La sentencia se basa en que las telecomunicaciones por cable se producen por medio de redes que no excluyen la existencia de otras redes, como ve nía siendo el caso de la televisión hertziana. De este modo, el derecho individual a la comunicación prevalece sobre el derecho del gobierno de asignar frecuencias limita das del espectro.

15 GIL (1996).

16 «Lo que sí está claro es que el Tribunal Constitucional presta una protección jurisdiccional bastante amplia a los actuales operadores; a la Administración Pública y al Legislativo ya no le queda más remedio que tener en cuenta las decisiones del más al to tribunal de este paíss (GALD ÓN, 1994: 33).

17 El Ayuntamiento de Barcelona hace público en el Boletín 0 ficial de la provincia del 29 de marzo de 1995 un «concurso para la utilización del dominio público local de Barcelona para la construcción y expansión de una red piloto de comunicaciones por cable... con carácter experimental». El plazo para la presentación de propuestas vence el 15 de mayo, prorrogándose después hasta el 30 de junio debido al enfrenta miento entre los dos niveles de gobierno: «El Ministerio jugó fuerte y el 1 de junio impugnó la convocatoria en vía contencioso administrativa, lo que hizo que la mayoría de los licitadores retiraran sus ofertas. D urante el verano se desarrolló una intensa actividad negociadora que dio como resultado la adjudicación, el 23 de septiembre, del concurso a la sociedad «Cable y Televisión de Cataluña» y el desistimiento del re curso contencioso por el M inisterio a cambio de participar en el proyecto técnico ne cesario para iniciar el cableado, según el convenio firmado por Borrell y $\mathrm{M}$ aragall el día 3 de octubre. El primer accionista de la empresa concesionaria es U.S West, con el 25 por 100, seguido por la Caixa de Catal uña con el 20 por 100, REDESA con 20 por 100, CABLEUROPA con 20 por 100 y, en menor proporción TISA (La Vanguardia, 7,5 por 100) y Catalana d'Iniciatives (Generalitat y Ayuntamiento, 7,5 por 100)» (Ló Pez Rodó, 1995: 5).

18 La doctrina de los tribunales del nivel autonómico es también un ejemplo del cambio institucional que supone el desarrollo del nivel regional sobre los otros dos niveles de autoridad.

$19 \mathrm{El}$ cambio institucional que supone la incorporación de los tribunales como esferas autónomas de autoridad reguladora viene acompañado de debates ilustradores sobre el tema. Así, los comentarios publicados por los juristas en libros y revistas especializadas contribuyen a establecer puntos críticos de controversia (SAnz León y M ARTiNEZ ALONSO, 1996).

20 En un primer momento, los gobiernos de Adolfo Suárez y Leopoldo Calvo Sotelo tienen como único objetivo la normalización democrática. Se trazan planes de reforma económica, pero su implementación no alcanza todos los puntos, como por ejemplo la empresa pública, bajo cuya rúbrica se encontraban filiales participadas de Telefónica, y parte de la propia compañía. D e acuerdo con el responsable del diseño del programa de reformas, Enrique Fuentes Q uintana, la intención prioritaria es fre nar la crisis económica con el objeto de llegar al consenso necesario para adoptar una Constitución. La percepción desde la presidencia era la de que o bien las reformas eran implementadas o no habría consenso sobre una constitución. H ay una crisis económica crítica, que puede llevar a la suspensión de pagos o a un elevado índice de inflación bajo cuyos efectos hubiese sido imposible elaborar una Constitución (Fuentes Q Uintana 1996: 260 - entrevista de Andreu M issé con Enrique Fuentes Q uintana, vicepresidente del gobierno durante el primer período de gobierno del presidente Suárez- , en M emoria de la Transición, M adrid: Taurus. pp. 259-265).

21 «La inflación bajó pronto de un 40 por 100 a un 20 por 100. En febrero de 1978 el nivel ya era del 18 por 100, y el año terminó con un índice del 14 por 100. Sin embargo una parte sustancial de las reformas fiscales fue detenida por los intereses existentes, y la reforma de la empresa pública fue pospuesta» (FuEN TES Q UIN TANA, 1996: 262).

22 En los primeros años de la década de 1980, el protagonismo activo del monopolio en la dirección del sector industrial de la tecnología de la información ha alcanzado su cenit. Telefónica llega a contar con participación efectiva en las cuatro empresas proveedoras de equipamiento de telecomunicaciones del país. Con una participación del 21,4 por 100 en Alcatel, del 80 por 100 en Amper y la promoción activa de la localización en España de Fujitsu, y ATT. La inversión de ATT M icroelectronica sefinanció en un 80 por 100 con capital de Telefónica y ayudas públicas de todo tipo. Fujitsu tuvo un 60 por 100 de financiación similar (G Am ELLA et al., 1991: 426).

23 D os gabinetes ministeriales tienen visiones enfrentadas sobre la conveniencia de las relaciones entre Telefónica y sus subsidiarias, Industria y Economía. El M inisterio de Economía se decanta por la estabilización económica y los recortes. Para el M inisterio de Industria, Telefónica es la piedra angular de la política industrial en el sector. En la política de liderazgo activa del monopolio y el Ministerio de Industria, este último asume en 1985 la política de establecimiento de multinacionales capaces de aportar capacidad tecnológica en el marco de un plan industrial (PEIN I).

24 La tran sferencia del holding y la venta de participaciones en empresas del sector también marcan un punto de inflexión básico en la política de la empresa.

25 El País, 1985, 23 de febrero, p. 44.

26 La política de contención de gastos había comenzado ya en 1983, (crecimiento cero del empleo, enfoque selectivo de las inversiones, contención de gastos generales y coyuntura favorable de restricción salarial fruto del Acuerdo de la M oncloa). Sin embargo, se había producido un incremento del recurso al crédito y los préstamos. D esde 1987 el crecimiento de la inversión de Telefónica comienza a superar el de la economía española. Este crecimiento (23 por 100 en 1987 a 63 por 100 en 1989, datos de $G$ am ELLA et al., 1991), ya viene impulsado por políticas de autofinanciación por la venta de empresas de grupo, subidas de tarifas y emisión de acciones en bolsas internacionales. En 1991 el monopolio ha conseguido elevar la tasa de autofinanciación desde el 41,9 por 100 con previsiones de superar el 100 por 100 a mediados de los noventa (GAm ELLA et al, 1991: 427).

27 Frankfurt, Tokio, París, Zurich y N ueva York.

28 El País, 1985, 31 de enero, p. 43; El País, 1989, 11 de septiembre, p. 10.

29 En este sentido responde sin anticiparse, al aumento inesperado de la demanda de servicios de telecomunicaciones, que se produce a caballo de la recuperación económica a mediados de los años ochenta. La demanda de líneas y su grado de utilización se doblan entre 1985 y 1989 en el mercado doméstico.

30 Chile, Argentina, Venezuela, Puerto Rico y Perú, en un principio.

31 En este período, el Director General de Telecomunicaciones, responsable de la regulación, actúa también como D elegado del Gobierno en el consejo de administración de Telefónica.

32 Estas agencias eran la Junta N acional de Telecomunicaciones y Gabinete de 0 rdenación de las Telecomunicaciones, Instituto de Estudios de Transportes y Comunicaciones, Dirección General de Correos y Telecomunicaciones, Telefónica, Ente PúbliCo RTVE, Dirección General de Aviación Civil, Dirección General de Marina $M$ ercante, M inisterio de D efensa y Dirección G eneral del M inisterio de Comunicación Social (LeRA LASO, 1986: 82).

33 Entrevista con Reinaldo Rodríguez Illera, Director General de Telecomunicaciones y vicepresidente del CAT.

34 Consejo que no se establece hasta que por Real D ecreto 970/1991 de 14 dejunio, se especifica su composición. Su composición incluye a representantes de los ministerios, las comunidades autónomas, los ayuntamientos, la industria de equipos de telecomunicaciones, los importadores y distribuidores, proveedores de servicios de tele- 
comunicación, usuarios, así como los sindicatos más representativos del sector. En 1992 (Real Decreto 1398/1992 de 20 de N oviembre), se amplía su representación incluyendo a representantes de la asociación de ingenieros de telecomunicaciones, re presentantes de los servicios de radio y televisión a nivel regional y local, y representantes de empresas que ofrecen servicios de telecomunicaciones de valor añadido. El CAT da su opinión al gobierno cuando se le requiere.

35 Entrevista con Reinaldo Rodríguez Illera.

36 Es más, la exclusión por medio de las prerrogativas del Estado prevaleceincluso actualmente, al ser el Estado el garante de la competencia.

37 Por la Ley 32/1992 de 3 de diciembre.

38 «U na de las novedades más importantes de la ley es la creación de un Fondo $\mathrm{Na}$ cional del Servicio Universal de las Telecomunicaciones, que tiene por finalidad ga rantizar la financiación del servicio universal, y cuya gestión es encomendada a la Comisión del Mercado de las Telecomunicaciones» (SANZ, 1999: 124).

39 Según la abogada ArPón De M en díviL (1995: 2), el Acuerdo potencia «la adaptación de Telefónica a las condiciones de competitividad e internacionalización del sector».

40 Por ejemplo, las concesiones para la transmisión de datos mediante conmutadores de paquetes o circuitos se apoyan en una definición de servicio de provisión de datos muy amplia, al incluir cualquiera de los que se prestan entre puntos de terminación de redes, en lugar de referirse sólo a aquellos que se prestan entre dos puntos terminales de la red pública conmutada (C ommunication by the Commission to the European Parliament and the Council on the Status and Implementation of D irective 90/388/EEC. Com(95) 113 final, p. 7).

41 Este proyecto faculta a Telefónica para ofrecer servicio de cable nueve meses después de que comience a prestar tales servicios una segunda empresa autorizada. Esta ventaja de emitir con anterioridad para un segundo operador podría interpretarse como una discriminación positiva para situar en igualdad de condiciones a dos competidores desiguales. Sin embargo, la igualdad es sólo aparente. M ientras que Telefónica puede proveer con servicios mediante cable en nueve meses, el segundo operador no está facultado para prestar los servicios que ofrece tradicionalmente $\mathrm{el}$ monopolio hasta el 1 de enero de 1998 (LAVILLA RUBIRA, 1995, ABC, 31 de octubre de 1995, p. 30).

42 «Es lo que ha permitido que entre los años 1996 y 1998 se hayan otorgado conce siones y derechos exclusivos, tanto en cable como en telefonía fija, permitiendo la aparición y el posicionamiento de algunos operadores de telecomunicaciones antes de la apertura total del mercado» (GarCíA-BRAGAD o y CLOTET, 1998: 292).

43 Término acuñado en instancias europeas para definir objetivos de políticas cuyo objeto es la difusión de las nuevas comunicaciones en la sociedad de la UE. Ver Antonio Alabau (1998) La Unión Europea y su política de telecomunicaciones. En el camino a la sociedad dela información.

44 M inisterio que estaba relativamente vacío de contenido desde que se produjeron las transferencias de competencias a Bruselas, y cayó en desuso la política industrial implantada en España por el régimen franquista a ráiz de la crisis económica de 1973.

45 Ver Real D ecreto-Ley 7/2000, de 23 de junio, deM edidas Urgentes en el sector de las Telecomunicaciones; Real D ecreto 3456/2000, de 22 de diciembre, por el que se aprueba el reglamento sobre condiciones de acceso al bucle local de abonado; aś como la 0 rden del M inisterio de la Presidencia, de 30 de enero de 2001.

46 EI CIECAT data de 1991, aunque se registra oficialmente como asociación el 26 de enero de 1993. Esta asociación se crea como respuesta al incremento de complejidad en el mercado y sus objetivos son facilitar la incorporación de nuevas tecnologías, como la multimedia y responder mejor a la sofisticación de software, reduciendo los costes (Adell y Cavero, 1997: 96). Los objetivos que busca el CIECAT cuando se constituye en asociación dan cuenta de la estrategia de las empresas miembros, como grupo y en relación a otros (ADELL y CAVERO 1997: 96-97): El intercambio de experiencias y la colaboración, la normalización de standards proponiendo normas de rango nacional e influyendo en las internacionales, la formación de repre sentantes de empresas, así como la mejora de la calidad y la innovación.

47 En este último sentido, M ONTERO y SOUTO (1999: 6-7) aportan evidencia de que la relación entre esferas de autoridad a distintos niveles resulta conflictiva. Así, la C omisión Europea se dirige oficialmente al Servicio de $D$ efensa de la Competencia, sin informar a la CM T, solicitando información sobre concentraciones de empresas de telecomunicaciones, como el caso de Cable i Televisió de Catalunya (Asunto IV/M .1022, C able i Televisió de Catalunya, D ecisión de 28-1-98). La reacción de la
CM T es un Acuerdo de su Consejo de 27 de noviembre de 1997: «no parece que se haya respetado en sus justos términos el procedimiento establecido para comunicar a los Estados miembros... [la CM T ] es el órgano especializado dentro del Estado español en la salvaguardia de los principios de libre competencia y no discriminación en el mercado de las telecomunicaciones» (cita de M ONTERO y Souto 1999: 6). «La Comisión Europea, sin embargo, parece confirmar que mantendrá comunicación oficial de sus actividades únicamente con una autoridad nacional, aquella que aplique directamente las normas de defensa de la competencia... por lo que la CM T deberá solicitar a los órganos nacionales de la competencia la comunicación de las acciones de la Comisión Europea» (M ontero y Souto, 1999: 7).

48 Se crea para asegurar el cumplimiento de la primera ley sobre la competencia en España, Ley de Represión de Practicas Restrictivas de la Competencia, 110/63. El marco institucional creado en 1963 para inducir la liberalización de la economía en España estuvo influido por el contexto internacional. Los Estados U nidos presionan a España para que abandone las prácticas monopolísticas y proteccionistas, insistiendo en que se introduzcan prácticas para promover la competencia y la productividad. $49 \mathrm{El}$ Tribunal envía comentarios al gobierno sobre aspectos relativos a la competencia de nuevos proyectos de ley. También se encarga de estudiar y remitir propuestas para modificar leyes ya existentes basándose en la experiencia adquirida a nivel nacional y de la Unión Europea. Además de su función como institución consultiva del gobierno, el Tribunal puede imponer multas que pueden superar los 900.000 euros (hasta 150 millones de pesetas), y que pueden incluso incrementarse hasta un 10 por 100 de los beneficios declarados el año fiscal anterior a aquel de la decisión adoptada por el Tribunal.

50 El mismo Tribunal reconoce que antes de 1989 la unidad técnica contaba con pocos funcionarios, tres o cuatro, y éstos no eran especialistas en temas de competencia. Es más, los funcionarios se encontraban en minoría en un medio hostil a ideas que eran novedosas a principios de los 1960s. El Tribunal autoevalúa negativamente su labor en los primeros años de su creación: «El Tribunal ha sido un organismo al que se accedía a una edad relativamente avanzada, y cuyo puesto se mantenía hasta que se agotaba toda posible renovación, cuando se contaba con 75 años de edad. Esta característica del Tribunal ha influido en su natural eza y sin duda, ha afectado la actitud que adoptó y cómo sus responsabilidades se llevaban a cabo.» (Tribunal de D efensa de la Competencia. 1989. La Libre Competencia en España 1986-1988, Madrid, p. 14).

51 La ley de 1989 capacita al Tribunal para actuar sobre casos de competencia desleal que distorsionan el libre mercado. Prohíbe también el abuso de posición dominante, cláusula que se aplica además a los monopolios establecidos por la ley. El 17 de diciembre de $1999 \mathrm{El}$ pleno del Congreso aprueba una nueva Ley de D efensa de la Competencia con los votos a favor de PP y CiU, y los votos en contra del PSOE e IU. PN V y el Grupo M ixto se abstienen. La ley se tramita por el procedimiento de urgencia, y en su tramo final, el 11 de noviembre use encontró con una sentencia del Constitucional que declaraba inconstitucionales seis artículos del anterior texto. Como quiera que el texto aprobado ayer se basa en la misma filosofía, el Gobierno introdujo este mes una enmienda adicional en la que se compromete a adaptar en el 2000 los artículos a la doctrina del Constitucional» (El País, 17 de diciembre de 1999, p. 84). Una nueva ley de la competencia se aprueba en enero de 2002.

52 Para una relación del papel destacado del TDC en el sector de las telecomunicaciones, así como de las disputas institucionales con otras instituciones del nivel de autoridad del Estado central, como la CM T, ver M onTERo y BRoKeLmann (1999: 156168): «C onflictos relacionados con la aplicación de las normas de la competencia».

53 El Tribunal lo componen ocho miembros propuestos por el M inisterio de Economía por su probado prestigio entre abogados, economistas y otros profesionales con más de 15 años de experiencia. Los miembros son confirmados en sus puestos por el gobierno, y son elegidos por un período de 6 años con posibilidad de renovación. M iguel Ángel Fernández, ex presidente del Tribunal de D efensa de la Competencia critica al gobierno del PP por variar por decreto-ley la composición del tribunal, lo que ataca su independencia. También critica la misma reforma administrativa en marcha, que hace depender al SDC jerárquicamente de los políticos, y fortalece este organismo frente al Tribunal (El País, 15 de diciembre de 1999).

54 El Servicio de D efensa de la Competencia puede tomar medidas basándose en la Ley 16/1989. Se encarga de supervisar la aplicación de decisiones basadas en la Ley 16/1989. Su posición es reforzada en la reforma de la ley de 1989 de diciem- 
bre de 1999, y su composición puede estar ligada más de cerca al gobierno desde esta fecha.

55 Este registro mantiene la documentación referente a decisiones y recomendaciones del Tribunal de $D$ efensa de la Competencia. También recoge la información referente a concentraciones empresariales.

56 La primera decisión se adopta en octubre de 1993. La sentencia condena a Telefónica por obligar a la compra de cierto tipo de terminales telefónicos por medio de una tasa de conexión cargada en el primer recibo telefónico, a pesar de que el contra to contemplaba el simple alquiler de los terminales. El Tribunal consideró que la tasa de conexión equivalía al precio de compra de los terminales, lo que suponía un abuso de la posición dominante de una empresa con posición de monopolio. Una segunda decisión se toma en febrero de 1995. En este caso se impone una multa de 745.000 euros (124 millones de pesetas) a Telefónica por retrasar la conexión a sus terminales a la empresa 3C Communications de España. La empresa 3C Communications tra taba de proveer servicio telefónico por medio de tarjeta de crédito en los aeropuertos. La calificación del Tribunal del servicio telefónico pagado mediante tarjeta de crédito como un servicio de val or añadido desarmó la pretensiones de Telefónica de conside rarlo como servicio básico - lo que hubiera significado la reserva de la provisión a su monopolio según el art. 2.1. de la Ley 32/1992-.

57 El Tribunal considera que las tarifas telefónicas en España son las más altas de toda la OCDE. Como consecuencia de este hecho, la economía española no puede ser competitiva. En opinión del Tribunal, una sola compañía no puede abarcar todo un sector de la economía, cuando éste ha crecido tanto en complejidad como lo ha hecho el de las telecomunicaciones. El Tribunal estima que una sola compañía tampoco puede aportar las inversiones necesarias para el desarrollo del sector, y que un mercado abierto a la competencia, a la vez que ofrece oportunidades para la diversificación de las inversiones, atraería mas capital que un mercado en régimen de monopolio.

58 EI TDC condena «a principios de 1999 a Telefónica por la imposición de condiciones de exclusividad a sus clientes en el mercado de arrendamiento de circuitos, así como por la imposición de una cantidad mínima de circuitos, a fin de garantizar que la totalidad de la contratación de sus clientes se realizase con Telefónica a fin de alcanzar dichos mínimos y beneficiarse de las condiciones ofrecidas por el ex-monopolista [Tribunal de D efensa de la Competencia, Resolución BT/Telefónica de 21 de 3nero de 1999, Expediente 412/97)]» (M ON TERO y So UTO, 1999: 9).

59 Para aspectos administrativos relacionados con la CM T ver la relación de M ONTERO y BROKELMAN N (1999: 120-148).

60 Como muestra de ello, el presidente del Tribunal de D efensa de la Competencia hace declaraciones públicas intentando salvaguardar funciones de la institución que representa sobre el sector de las telecomunicaciones: «La cooperación es necesaria entre autoridades de defensa de la competencia e instituciones de vigilancia y regula ción... El proyecto de ley de telecomunicaciones explicita claramente esta coordina ción entre instituciones en la disposición adicional 7, cuando dice que el ejercicio de las funciones de la Comisión se realizará con pleno respeto de las competencias que la Ley de D efensa de la Competencia atribuye a los órganos de defensa de la compe tencia. De esta forma se salvan las dos instituciones, se salvan sus competencias» (Amadeo Petitió, Madrid, 8 de octubre 1997).

61 Declaraciones de Florenci Bach, 8 de octubre 1997.

62 En España, el M inisterio de Fomento opta por el concurso para adjudicar la 4a licencia de telefonía U M TS al grupo Xfera por 516 millones de euros (86.000 millones de pesetas). Gran Bretaña y Alemania optan por la subasta y obtienen 6,5 billones de pesetas y 8,4 billones respectivamente, diferencia que origina críticas caústicas a la gestión del gobierno español.

63 En este punto resulta interesante recordar la posición escéptica de M artin Shapiro (1998, entrevista) cuando recuerda que Comisión significa literalmente comisión, al udiendo indirectamente a la teoría de captura por parte de los grupos económicos de las $C$ omisiones que les regulan.

64 Declaraciones de Florenci Bach, miembro de la CM T. 8 de octubre 1997.

65 OFTEL se crea por la Telecommunications Act de 1984 y está dirigida por un Director General de Telecomunicaciones, elegido por un período fijo, y que solo puede ser retirado de su cargo bajo circunstancias especiales (H ULSINK, 1996: 194). Es el Director $G$ eneral quien se encarga de asegurar que se cumplen las condiciones contenidas en las licencias de los operadores, y cuenta con total discreción para impugnar licencias. El resto de los miembros de O FTEL, hasta 160, son funcionarios. Los fon- dos para sufragar la agencia proceden del Parlamento, si bien los costes son cubiertos por el precio de las licencias otorgadas, que en el caso de grandes operadores están en proporción con las ganancias. La estructura administrativa externa de OFTEL se compone de comités consultivos regionales y de intereses específicos: «Comités consultivos de telecomunicaciones (Inglaterra, Escocia, Irlanda del N orte y Gales), dos comités representando los intereses de pensionistas y personas con discapacidades, así como las pequeñas empresas; además se suma la obligación del D irector General de consultar a representantes de los grandes usuarios de telecomunicaciones y de los consumidores locales) (H ULSINK, 1996: 194).

${ }_{66}$ C.D. Foster, cercano al proceso político del diseño del regulador escribe: «fue una decisión política que British Telecomm no fuese regulada por ministros. En su lugar, la analogía sugería que hiciese el trabajo el D irector $G$ eneral de Comercio, que fue presionado para aceptar. Sin embargo, éste decidió que tenía suficientes funciones que desempeñar, de modo que se inventó un puesto que diese la impresión de que era asumido por un especialista, el Director General de Telecomunicaciones) (FosTER, 1992: 124).

67 Analizando el proceso de reforma de las telecomunicaciones en Gran Bretaña, H ULSINK, (1996: 343), afirma: «Puede definirse como improvisado y orientado a las empresas, con objetivos a corto plazo. El Libro Blanco de 1982, que expone el controvertido plan para liberalizar y privatizar British Telecommunications, contenía solo un breve comunicado ministerial de unas 5 páginas y algo de información que apoyaba tales decisiones. El nuevo régimen regulatorio fue en gran parte desarrollado paso a paso, de manera improvisada. La creación de OFTEL no aparecía en el plan original de 1981 y fue considerada solo un año más tarde, cuando la transición desde el monopolio a un entorno de mercado no ocurría de manera automática y necesitaba ser dirigida por una agencia reguladora».

68 Su modelo es poco participativo, predominando las negociaciones informales entre el regulador y la industria. La falta de transparencia está reforzada por la ausencia de obligación de consultas públicas o de estar abierta al escrutinio público. Tampoco existen muchas posibilidades para presentar recursos, mientras que el poder se concentra en manos del Director General (H ULSINK, 1996: 194).

69 «La Comisión fue nombrada enteramente por el Gobierno, pero respetando un cierto sistema de reparto de formaciones políticas. Tal y como está configurada en 1997, el PP propone a tres de sus siete miembros: el presidente, José M aría Vázquez Q uintana, y otros dos miembros, la abogada del Estado G loria Calvo D íaz, y el exconsejero deTelefónica, José M ontes Fernández. Por el PSO E fueron designados el ex-presidente de Retevisión, M iguel Ángel Feito y Reinaldo Rodríguez Illera, ex Director $G$ eneral de Telecomunicaciones, CiU propuso a Florenci i Bach (vicepresidente de la Comisión), y el PNV a Juan José Zubeldia». El País, jueves 13 de marzo de 1997, p. 30.

70 El resultado es una cúpula multipartidista y variada a finales de 1999: José M aría Vázquez Q uintana (PP), Florenci Bach (CiU), Gloria Calvo (PP), José M ontes (PP), Miguel Ángel Feito (PSOE), Reinaldo Rodríguez (PSOE), Juan José Zubeldia (PN V), I gnacio M. Fernández (Izquierda Unida), Rosendo Reboso (C oalición C anaria) (Expansión, viernes 10 de diciembre de 1999).

71 Así lo atestigua el trabajo de Jacint JORD ANA (1999b: 245): «Telefónica, por una parte, y algunos grupos mediáticos como Planeta o Prisa, por otra, han buscado fórmulas para extender sus actividades en áreas conexas, como las nuevas ofertas televisivas o el cable, donde en ocasiones coincidían entre sí. Junto a estas empresas ya presentes previamente en el macro-sector, la liberalización facilitó la entrada de nuevos actores empresariales deseosos de participar en estas actividades. Así, tanto grupos multinacionales de las telecomunicaciones, como British Telecom, France Telecom, o Stet, y grupos empresariales y financieros españoles, como Endesa, Unión Fenosa, BCH, Banco Santander o La Caixa entre otros, apostaron por comprar e invertir en el sector, consiguiendo participaciones significativas en las empresas clave y también espacios de influencia en la configuración de los nuevos desarrollos empresariales»,

72 Dirección General de Telecomunicaciones y Secretaría General de Telecomunicaciones.

73 El tema de la base fiscal ha resultado muy controvertido en Europa, donde las ansias recaudatorias de los gobiernos en el año 2000 ascienden a 120.000 millones de euros en concepto de licencias para la explotación de telefonía móvil de tercera generación (UM TS), una tecnología que en 2003 aún no ha arrancado comercialmente. 


\section{Bibliografía}

Alabau, A. (1998). La U nión Europea y su política de telecomunicaciones en el camino a la sociedad dela información. Ávila: Fundación Airtel M ovil.

Adell Hernani, J.A., Cavero Clerencia, J.M . (1997). «a colaboración empresarial parala calidad en telecomunicaciones: el CIECAT», BIT, n. 101 (Ene-Feb). 96-99.

ARPON DE M EN DíVIL, A. (1995). «Telecomunicaciones en España: situación legal y perspectivas de negocio». Comunicación presentada al Primer Congreso Español de Derecho de lasTelecomunicaciones. Madrid, 13-14 Diciembre.

Cawson, A., et al. (1990). Hostile Brothers. Competition and Closure in the European Electronics Industry. Clarendon Press: 0 xford.

GALD ON, E. (1994). «Posibilidades del cable en España a la luz de la experiencia internacional» Actas de la Conferencia Internacional sobre Televisión por Cable y Autopis tas dela Información, organizada por la Junta de Castilla y León en Salamanca, 22-23 Septiembre. 26-33.

Foster, Ch. D. (1992). Privatization, Public O wnership and Regulation of $N$ ational Monopoly. 0 xford: Blackwell.

Fuentes Q uintana, E. (1996). Entrevistado por Andreu M issé, M emoria dela Transción, M adrid: Taurus. 259-265.

Gamella, M., de la Puerta, E., Ayala, L., M atías, C. (1991). La Industria de las Tecnologías de la Información (1985-1990): España en el Contexto M undial. M adrid: Fundesco.

García Bragado, R., Clotet, M . (1999). «El proceso de liberalización de las tele comunicaciones y los municipios en $C$ ataluña», Políticas de telecomunicaciones en Es paña. JordanA, Jacint, SAN CHO, D avid, (Eds.). M adrid: Tecnos.

GIL, 0. (2002). Telecomunicaciones y Política en Estados U nidos y España: Construyendo M ercados. M adrid: CIS.

GIL, 0. (1994). «Structuring Telecommunication M arkets from the US and the Bra zilian Perspectives,» master thesis, U niversity of North Carolina at Chapel Hill, (M ay).

- - . (1995): «0 n W hether or not a critical juncture is taking place in Spanish politics. The Structuration of an Economic Sector and H ow the Roles of the Central and the Regional Governments are being Redefined» Propuesta de investigación empírica premiada bajo el programa Salvador de M adariaga del gobierno español en el Instituto Universitario Europeo, Florencia.

Gunther, R. (1996). «The Impact of Regime Change on Public Policy: the C ase of Spain», Journal of Public Policy. 157-202 (M ay-June).

H OLLIN GSWORTH, R. (1997). «T he Institutional Embeddedness of American Capitalism», Political Economy of M odern Capitalism: M apping Convergence and D iversity. Crouch, Colin, STREECK, Wolfgang (Eds.). London: Sage. 133-148.

LÓPEZ ROD Ó, L. (1995). «El proceso de elaboración de la regulación de las telecomunicaciones en Europa y en España». Comunicación presentada al Primer Congreso Espanol de D erecho de lasTelecomunicaciones, Madrid, 13-14 de D iciembre.

JORDANA, J. (1999). «El camino hacia la sociedad de la información: son útiles los planes y las políticas para su promoción?», Políticas de telecomunicaciones en Espa ña. JordanA, J., SANCH O, D., (Eds.). M adrid: Tecnos.

- - . 1997. Del M onopolio al M ercado: La Liberalizacion de las Telecomunicaciones en España (borrador).

LERA LASO, E. (1986). El futuro de lastelecomunicaciones espanolas: prospectiva y prevision tecnológica. M adrid: FU N DESCO.

M aravall, J. M. (1996). «C risis y Luces Rojas», M emorias de la Transicion, Ju LiÁ, Santos, Pradera, Javier y Prieto, Joaquín (C oor.) M adrid: Taurus. 467-472.

M CKeown, T. (1999). «T he Global Economy, Post-Fordism, and Trade Policy in Adavanced Capitalist States», Continuity and Change in Contemporary Capitalism.
Kitschelt, H., Lange, P., Marks, G., ST ephens, J. D. (Eds.). Cambrigde UK: Cambrigde University Press.

M ONTERO, J.J. (1999). «iSalvaguardia de la competencia o de los competidores? 0 tra visión de la política española de telecomunicaciones», Revista del $D$ erecho de las Telecomunicaciones e Infraestructuras de Red (RED ETI). Vol. 3. (Jun). 145-162.

M ontero, J.J., Brokelmann, H . (1999). Telecomunicacionesy Televisión: la Nueva Regulación en España. Valencia: Tirant M onografías, n. 118.

M Ontero, J.J., SOUto, L. (1999). «Telecomunicaciones y derecho de la competencia: de la desmonopolización a la competencia efectiva» La liberalización de las telecomunicacionesen un mundo global. Crem ADES, Javier, M AYOR-M EnÉnd Ez, Pablo. Madrid: La Ley-Actualidad.

N OLL, R.. (1987): «T he Political Foundations of Regulatory Policy», Congress Structure and Policy, M cCubbins, M athew D.; Sullivan, Terry (Eds.). N ew York: Cambrigde University Press.

O LIAS DE LIMA, B. (1999): «Las Comunidades Autónomas en un mundo globalizado», ponencia y debate en el $X$ Seminario Permanente de Administración y Economía Pública. D epartamento de Gobierno y Administración Pública, Instituto Universitario 0 rtega y Gasset. M adrid, 6 de M ayo.

PAstor, Carles. (1996). «C risis y Luces Rojas» M emorias de la Transición, Julía, Santos, Pradera, Javier and Prieto, Joaquín (Coor.) M adrid: Taurus. 533-540.

Petitió, Amadeo. (1993). «La política de telecomunicaciones en España. Situación actual». Los problemas de las telecomunicaciones en España (I). M adrid: Círculo de Empresarios. 91-108.

PrzeWORSKI, A. (1991). Democracy and the M arket. Cambrigde: Cambrigde University Press.

Q UINTANA, I. (1999). «Politica y sociedad: el lobby en Espana», ¿Existe sociedad civil en España?: Responsabilidades colectivas y valores públicos. SubIRATS, Joan (Ed.). M adrid: Fundación Encuentro (correo@fund-encuentro.org). 407-426.

Sanz León, A; Martínez-Alonso, J. L. (1996). «Estrategias, Actores y Políticas Públicas en el Proceso de Implementación de las Telecomunicaciones y la Televisión por Cable, EI C aso de Cataluña,» Trabajo presentado al II Congreso Español de Ciencia Política y de la Administración, Santiago de Compostela, 18-20 April.

SANZ, A. (1999): «D ebate político y marco regulador de las telecomunicaciones en España» Políticas de telecomunicaciones en España. J J RD AnA, Jacint, SAN CH O, D avid, (Eds.). M adrid: Tecnos.

Tribunal de D efensa de la Competencia. 1989. La Libre Competencia en España 1986-1988, M adrid.

Waverm AN, L., Esen, S. (1997). «European Telecommunications M arkets on the Verge of Full Liberalization» Vol. 11, n. 4 Fall 1997, págs. 113-126.

TOMÉ, B. (2001). «La política de desarrollo de la sociedad de la información en España». Revista de Estudios Económicos. n. 1 y 2. 3-24.

VÁzQuez Quintana, JoséM aría. 2001. «El papel de la Comisión del M ercado de las Telecomunicacioness. Revista de Estudios Económicos. n. 1 y 2. 23-38.

WeBer, M . (1978). Economy and Society: An Outline of Interpretative Sociology, G. Rотн y C. WITTICH. Berkeley: University of California Press.

\section{Entrevistas}

BACH, Florenci. Vicepresidente de la Comisión del M ercado de las Telecomunicaciones. Madrid, 7 octubre 1997.

Rodríguez Illera, Reinaldo. Director General de Telecomunicaciones. Madrid, 16 julio, 1996.

SHAPIRO, Martin. IUE, Florencia, 17 de febrero de 1998. 
